\title{
Flexible and Persistent Tool-using Strategies in Honey-gathering by Wild Chimpanzees
}

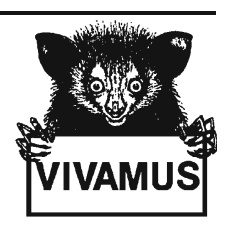

\author{
Crickette M. Sanz • David B. Morgan
}

Received: 22 August 2008 / Accepted: 18 February 2009 /

Published online: 12 March 2009

(C) The Author(s) 2009. This article is published with open access at Springerlink.com

\begin{abstract}
Several populations of wild chimpanzees use tools to raid bee nests, but preliminary observations of chimpanzees in the Congo Basin indicate that they may have developed sophisticated technical solutions to gather honey that differ from those of apes in other regions. Despite the lack of habituated groups within the range of the central subspecies, there have been several reports of different types of tools used by chimpanzees to open beehives and gather honey. Researchers have observed some of these behaviors (honey dipping) in populations of western and eastern chimpanzees, whereas others (hive pounding) may be limited to this region. Toward evaluating hypotheses of regional tool using patterns, we provide the first repeated direct observations and systematic documentation of tool use in honey-gathering by a population of Pan troglodytes troglodytes. Between 2002 and 2006, we observed 40 episodes of tool use in honey-gathering by chimpanzees in the Goualougo Triangle, Republic of Congo. Pounding was the most common and successful strategy to open beehives. Chimpanzees at this site used several tools in a single tool-using episode and could also attribute multiple functions to a single tool. They exhibited flexibility in responses toward progress in opening a hive and hierarchical structuring of tool sequences. Our results support suggestions of regional tool using traditions in honeygathering, which could be shaped by variation in bee ecology across the chimpanzee range. Further, we suggest that these chimpanzees may have an enhanced propensity to use tool sets that could be related to other aspects of their tool repertoire. Clearly, there is still much to be learned about the behavioral diversity of chimpanzees residing within the Congo Basin.
\end{abstract}

C. M. Sanz $(\square)$

Department of Primatology, Max Planck Institute for Evolutionary Anthropology, 04103 Leipzig, Germany

e-mail: sanz@eva.mpg.de

D. B. Morgan

Lester E. Fisher Center for the Study and Conservation of Apes, Lincoln Park Zoo, Chicago, Il 60614, USA

D. B. Morgan

Wildlife Conservation Society, Brazzaville, Republic of Congo 
Keywords Congo Basin Pan troglodytes troglodytes $\cdot$ serial tool use $\cdot$ tool set · video analysis

\section{Introduction}

The tool-using skills of chimpanzees are distinguished from those of other nonhumans by a combination of factors, including the use of $\geq 2$ types of tools to achieve a goal and flexibility in adapting tool strategies to new circumstances. Several populations of wild chimpanzees use tools to raid bee nests, but preliminary observations of chimpanzees in the Congo Basin indicate that these apes may have developed sophisticated technical solutions to gather honey that differ from those of apes in other regions. Differences in tool using strategies may reflect responses to different bee taxa, functional tactics for coping with target beehives, or socially transmitted traditions that differ between groups of chimpanzees (McGrew 1992). We attempt to precisely identify differences in toolusing strategies among populations by compiling reports from across the species range. This provides an informative context for presenting the first full descriptions of tool-using behaviors in honey-gathering by a population of the central subspecies of chimpanzee (Pan troglodytes troglodytes).

Chimpanzees typically gather the honey of African honey (Apini) and stingless (Meliponini) bee species that is stored in wax or resin vessels within the nest, but they also dip for the honey of carpenter bees (Xylocopa sp.) at some sites in West Africa (Table I). Nests of Apis may contain several kilograms of honey, whereas stingless bees rarely store $>1 \mathrm{~kg}$ (Roubik 1989). Bees have developed effective means of protecting their hives that most often involve the fortification and concealment of their nests. Different bee species show particular nesting habits, but there is also variation in nest building within species (Roubik 1989). Bee nests of Apidae are most often built in tree hollows or other preexisting cavities (Roubik 1989). Other bees may find lodging underground, in the forest canopy, or within the nests of other insects such as ants or termites. Certain bees also restrict or close the nest entrance when an intruder is detected. Another form of nest defense is to pursue or sting the intruder. Apis and Meliponini both have alarm pheromones that mark the hive raider so as to direct their conspecifics to the threat. The task of the honey-gathering chimpanzee is to overcome the defensive strategies of the bees themselves, breach the protective structure of the hive, and extract the honey and larvae.

Previous reports of chimpanzee tool use in honey-gathering describe several different tools and associated behaviors (Table II), which together are referred to here as tool strategies. Inserting a probe into a beehive to extract honey (dipping) is the most widespread tactic used by chimpanzees in honey-gathering (Ivory Coast: Boesch and Boesch 1990; Guinea: Ohashi 2006; Central African Republic: Fay and Carroll 1994; Gabon: Boesch et al. in press; Tutin et al. 1995; Republic of Congo: Bermejo and Illera 1999; Sanz and Morgan 2007; Tanzania: Izawa and Itani 1966; Uganda: Nishida and Hiraiwa 1982, Stanford et al. 2000, Watts 2008, Wrangham and Muller, pers. comm.). Chimpanzees may also manually detach an entire hive or a branch segment that contains a bee nest, and then use a dipping tool to extract the honey (Ohashi 2006). As reported from populations in Nigeria, 


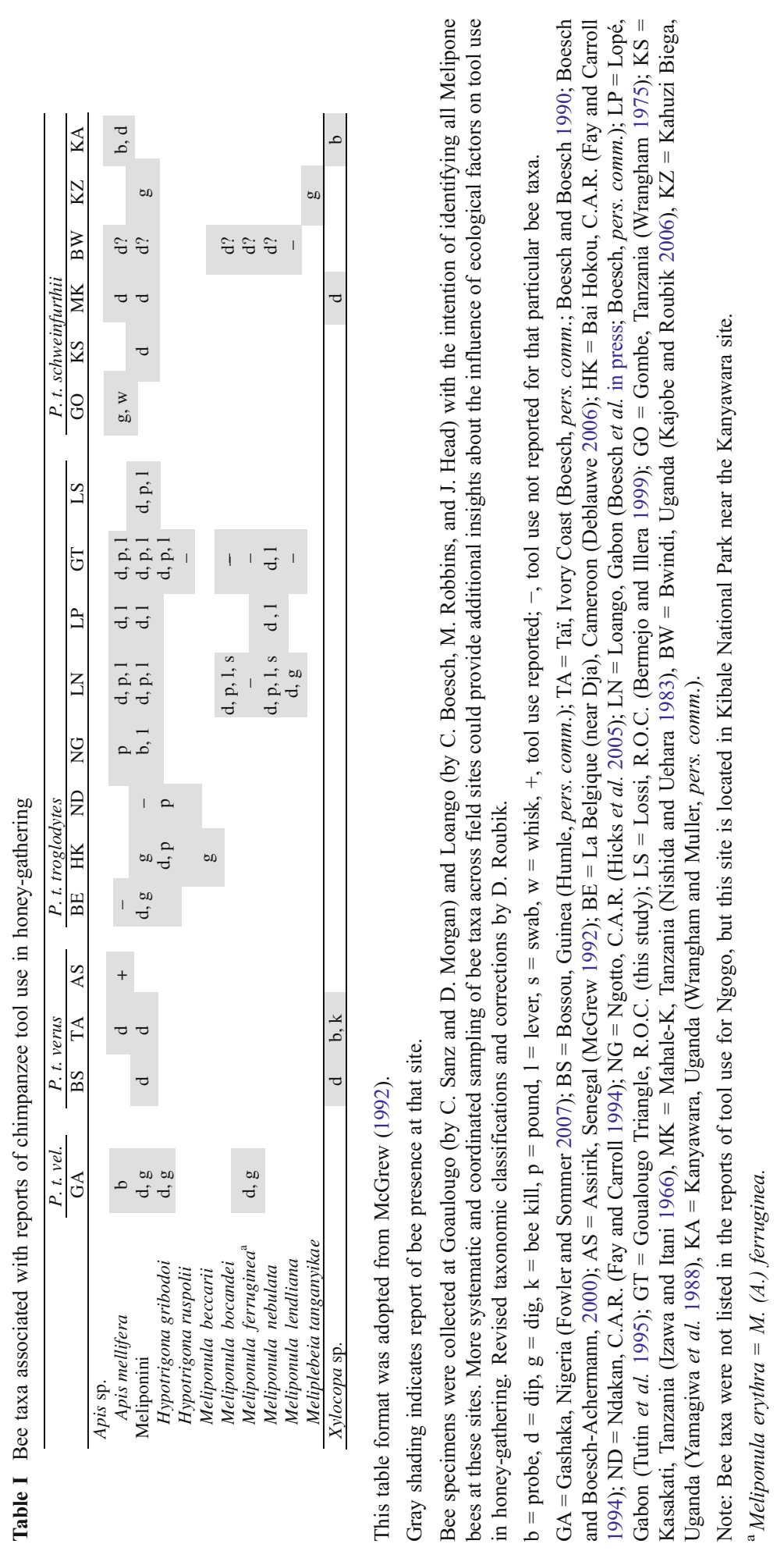




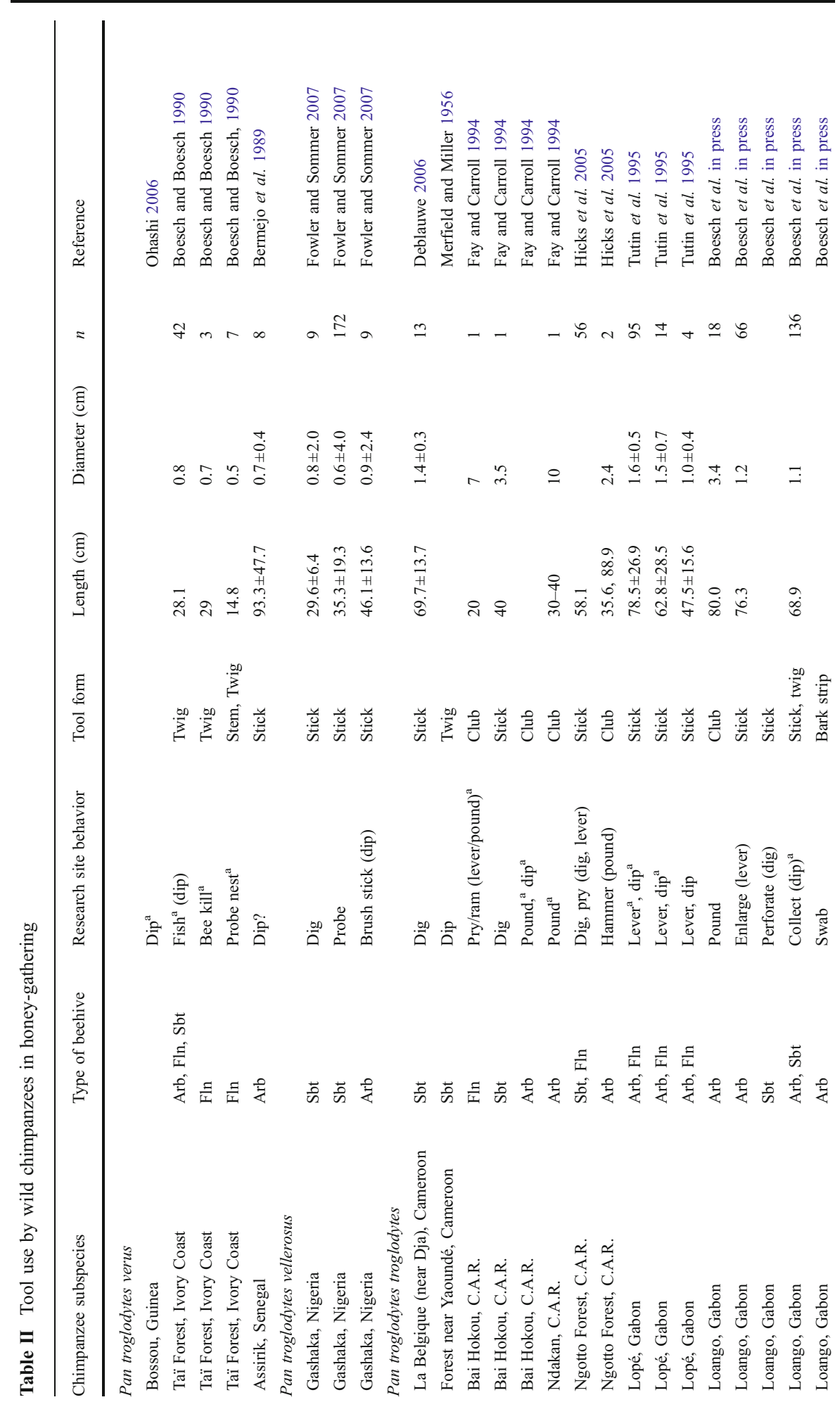




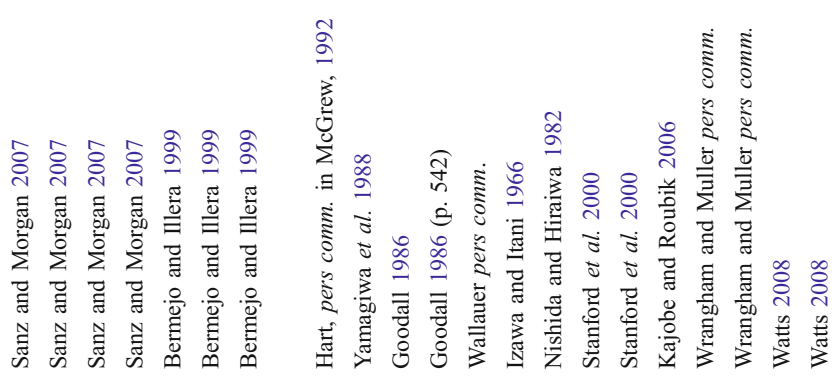

$\widehat{4}-4-4 c-$

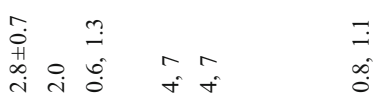

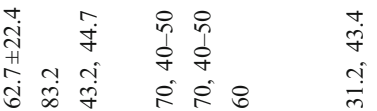

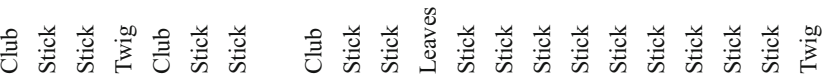

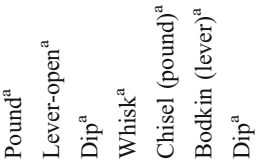

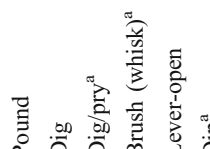

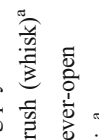

ㄷำ-

$\stackrel{-}{0} \stackrel{n}{0} \stackrel{0}{0}$

?

$8 \hat{\sim} \stackrel{\infty}{m}$

○

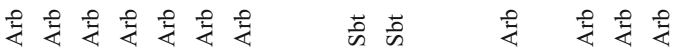

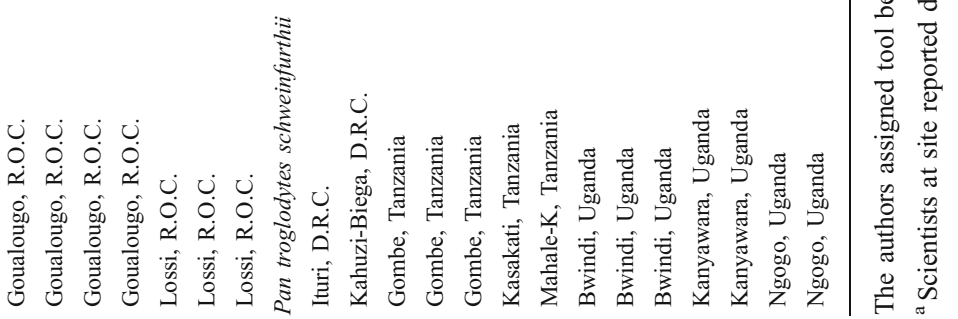




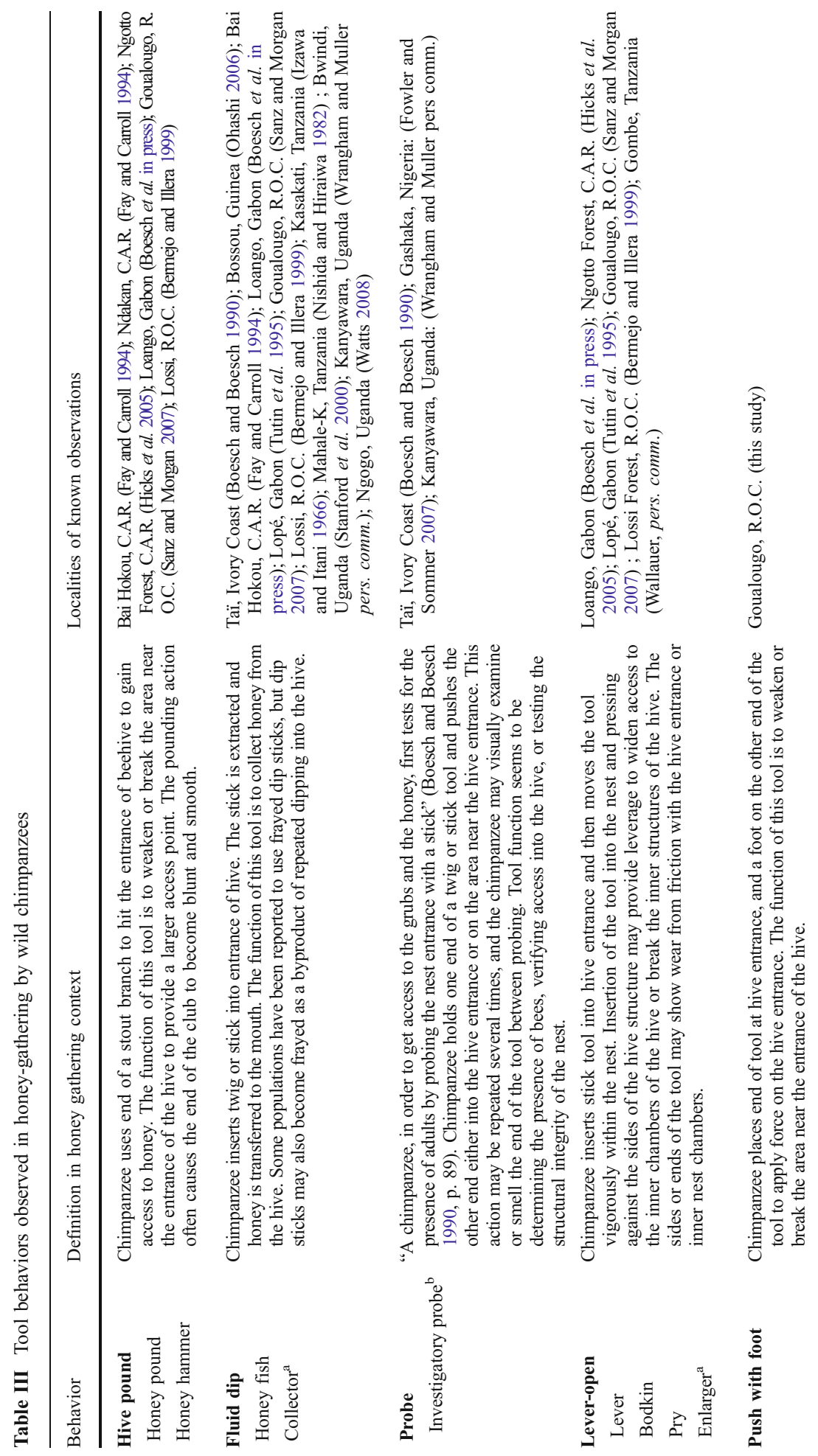




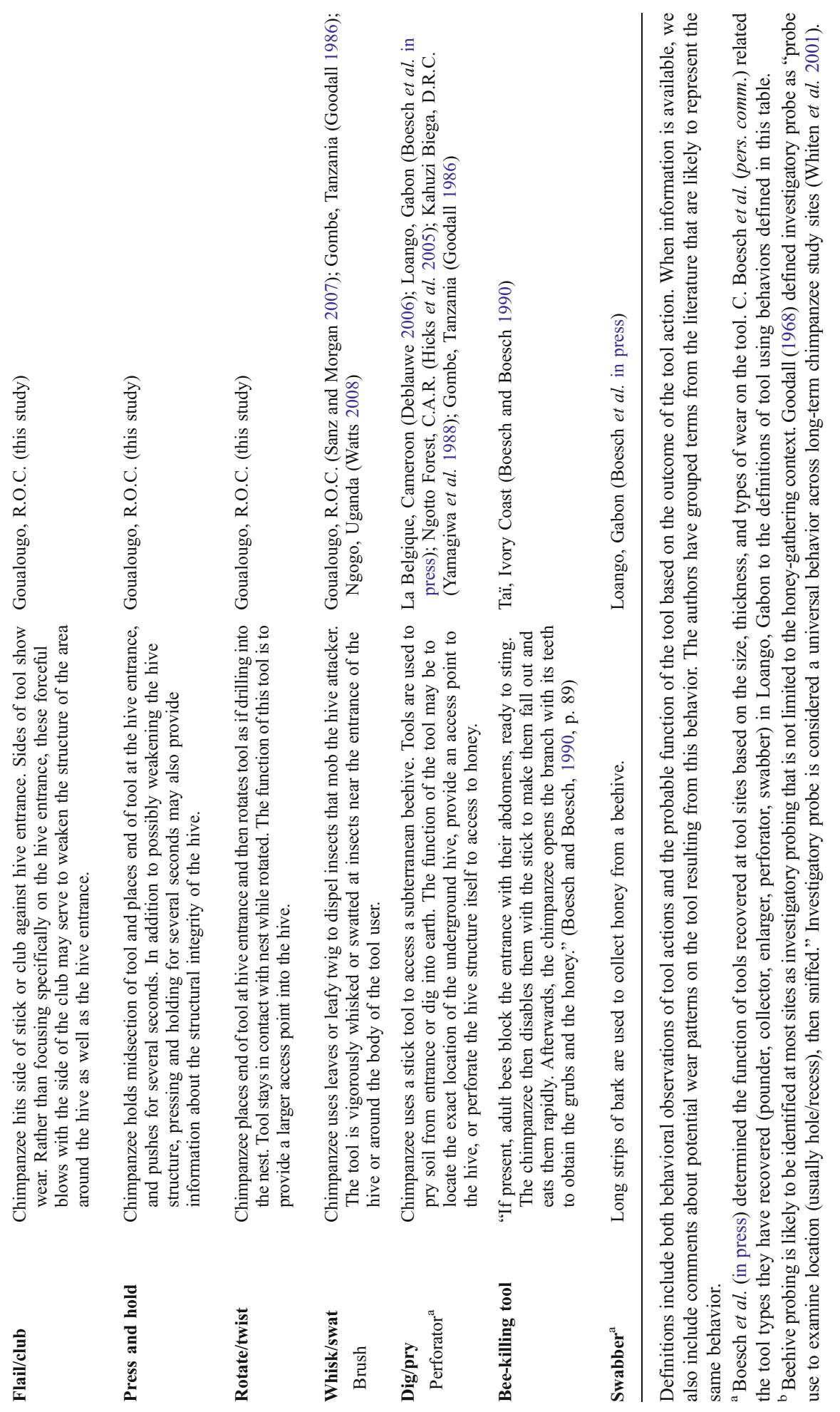


Cameroon, Central African Republic, and Tanzania, it is less common for chimpanzees to use a tool to dig or pry open subterranean hives (Deblauwe 2006; Fay and Carroll 1994; Fowler and Sommer 2007; Goodall 1986; Hicks et al. 2005). Researchers have observed chimpanzees using a tool as a lever to widen the access point to extract honey in Tanzania, Central African Republic, Gabon, and Republic of Congo (Bermejo and Illera 1999; Fay and Carroll 1994; Hicks et al. 2005; Sanz and Morgan 2007; Tutin et al. 1995; Wallauer, pers. comm.). Researchers have rarely observed pounding or hammering of beehives with the end of a large club to break the hive, but it seems exclusive to chimpanzee populations of the Congo Basin (Central African Republic: Fay and Carroll 1994, Hicks et al. 2005; Republic of Congo: Bermejo and Illera 1999; Sanz and Morgan 2007). Based on the geographical distribution of particular behaviors (Fig. 1), Hicks et al. (2005) hypothesized that there are regional honey-gathering traditions among wild chimpanzees. However, it is important to define tool-using behaviors more precisely so that observers can accurately identify similarities and differences between sites (Table III).

Although there are several published reports spanning $>50 \mathrm{yr}$, the available information on the honey-gathering by wild chimpanzees in central Africa has been limited to recovered tool assemblages or fleeting observations of unhabituated apes. We examined if previous suggestions of regional tool using traditions in honey-gathering are supported by direct observations of chimpanzees in central Africa. We analyzed video recordings of honey-gathering to define tool behaviors more precisely in this context and examine the plausibility of regional patterns of tool use. We also summarize information on the taxonomic composition of bee species to investigate if characteristics of the target hives may be shaping the honey-gathering strategies of wild chimpanzees across sites.

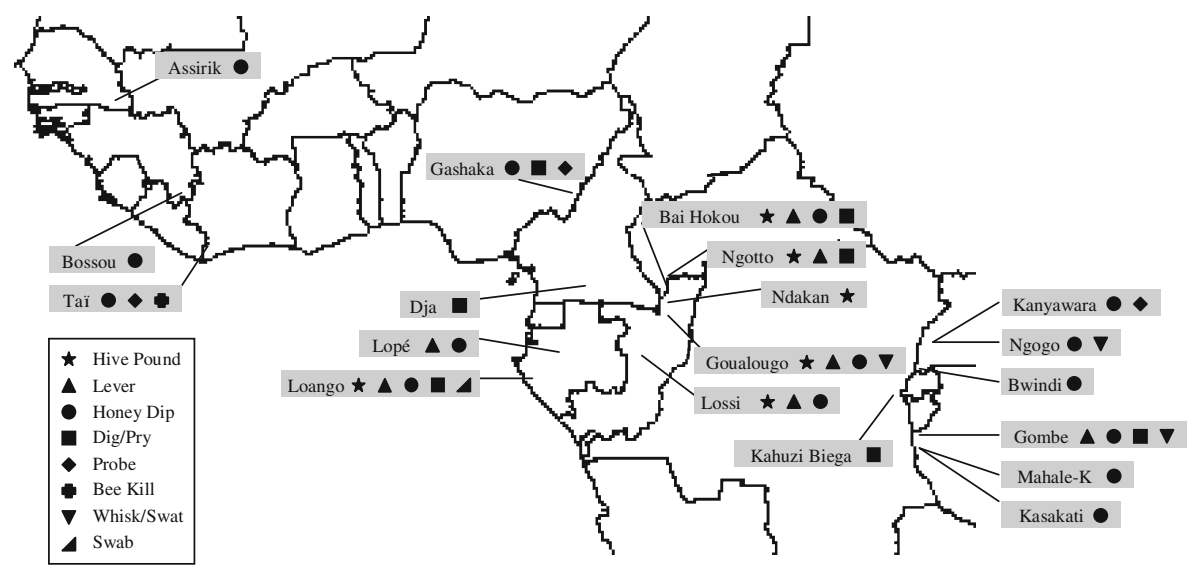

Fig. 1 Location of sites where chimpanzees use tools to gather honey. Symbols indicate the types of tool use at each site. 


\section{Methods}

\section{Study Site}

The Goualougo Triangle is located in the southern portion of the Nouabalé-Ndoki National Park, Republic of Congo. The study area covers $380 \mathrm{~km}^{2}$ of evergreen and semideciduous lowland forest, with altitudes ranging between 330 and $600 \mathrm{~m}$. The climate is transitional between the Congo-equatorial and subequatorial climatic zones. Rainfall is bimodal, with a main rainy season from August through November and a short rainy season in May.

\section{Study Population}

Between February 1999 and December 2006, we spent 88 mo in the Goualougo Triangle habituating and studying wild chimpanzees. We conducted reconnaissance surveys in several community ranges, but the majority of this time was concentrated on the semihabituated Moto community, which consists of 70 individuals, including immatures.

\section{Data Collection}

We documented chimpanzee tool-using behaviors via direct observation. For all instances of tool using behavior, we recorded the actor, type of object used, target of object, actions, context or goal of the tool-using behavior, and the outcome, which we used to define tool function. We recorded digital video of tool-using behaviors, duration of honey-gathering episodes, other chimpanzees present, and collected tools whenever possible. We recorded the location of each tool site, species of targeted beehive, materials used to make the tool, length, diameter, and any modifications to the raw materials. Tool measurements are reported in Sanz and Morgan (2007).

We also collected information about the height, size of the hive entrance, and other physical defenses of the target beehive. We adopted Roubik's (1989) classification of bee nesting biology, which includes digger bees, lodger bees, and mason bees. Digger bees excavate nests in the ground or other substrate. Lodger bees modify preexisting cavities. Mason bees construct nests that are not enclosed in a substrate.

\section{Video Data Analysis}

We archived data from digital video cassettes on external hard drive devices and converted them to MPEG files for review. We conducted video analysis using INTERACT Version 8.04 (Mangold 2006). We scored video recordings as tool-using episodes and bouts (Yamakoshi and Myowa-Yamakoshi 2004). An episode begins when the chimpanzee manufactures a tool or the first moment after which they are observed with the tool and ends when the tool is discarded or the task is abandoned. A bout begins when a chimpanzee uses a tool toward achieving a goal and ends when he/she attains the goal, begins using another tool, discards the tool, or pauses use of the tool for $>5 \mathrm{~s}$.

Using an ethogram of tool behaviors compiled from reports of honey-gathering at other sites (Table II), we scored tool using actions within video recorded episodes of honey-gathering. If we did not find an observed behavior on the list of existing 
definitions, then we operationally defined the novel behavior. We calculated Cohen's $\kappa$ to assess point-by-point agreement for tool actions (pound, dip, lever-open, push with foot, flail/club, press and hold, rotate/twist, whisk/swat) between Sanz and an undergraduate student assistant. We scored tool actions as events, and the interval width for accuracy was 5 frames. Interobserver reliability between 2 coders was $\mathrm{K}=$ 0.84 ( $n=23$ episodes), which is considered excellent (Fleiss 1981).

Elements are defined as functionally distinct behavioral units (Byrne and Byrne 1993; Byrne et al. 2001). To illustrate examples of multiple tool use, we graphed sequences of behavioral elements (including tool actions) as they occurred in honeygathering episodes. Sanz and Morgan (in press) define the essential elements and structures of several tool-using behaviors observed in the Goualougo Triangle chimpanzee population.

\section{Results}

Between 2002 and 2006, we observed 40 episodes of chimpanzees using tools to gather honey. We video recorded 30 of these episodes, representing 12 different individuals and subsequently analyzed them in greater detail. Chimpanzees in Goualougo most often used tools to open and raid arboreal Meliponini hives, but also targeted beehives located in fallen trees and subterranean hives. We often located beehives in areas that were difficult to access (Fig. 2), such as tree hollows, under the bark of living and rotting trees, and embedded in arboreal ant nests. The most common defenses of the bees were the structure of the hive and mobbing of the nest invader. Attempted beehive raids took place throughout the year in Goualougo, with no apparent seasonal trends. There were slightly higher frequencies of honey-gathering in April and from August to September, which coincides with environmental cues such as minimum temperature in the dry season which trigger flowering events that influence honey production by bees.

The average duration of honey-gathering observations was $19 \min (n=26$, which include the full episode), with successful episodes lasting $31 \mathrm{~min}$ on average and unsuccessful episodes $<5$ min on average. Chimpanzees were successful in obtaining honey in half $(52 \%)$ of our video-recorded observations. It was clear that they expended significant effort to open the hive and attain relatively small amounts of honey. The average number of tool using bouts in a successful honey-gathering episode was $14 \pm 15.7$ ( $n=14$ episodes), compared to only $2.67 \pm 2.74$ in 12 unsuccessful attempts. The ratio of tool actions to goals for honey-gathering chimpanzees showed that an average of 6 tool actions $(n=6$, average $=6.10 \pm 8.15$, range $=0.23-22.8$ ) were required for every taste of honey. The total number of tool actions ranged from 1 to $>1000$ beehive pounding actions in a single episode.

In total, we observed 8 distinct types of tool actions during video recordings of honey-gathering (Table III). Beehive pounding was the most common tool tactic, accounting for $94 \%$ of all tool actions and shown by 11 individuals (adults/subadults= 6 , juveniles $=5$ ). Fluid dipping accounted for $2.1 \%$ of tool actions and was exhibited by 5 individuals (adults/subadults $=3$, juveniles $=2$ ). Lever-opening comprised $1.8 \%$ of tool actions, and was shown by 7 individuals (adults/subadults $=4$, juveniles $=3$ ). Five individuals (adults/subadults $=3$, juvenile $=2$ ) exhibited a press-and-hold action, but this behavior comprised only $0.3 \%$ of tool actions. Two juveniles flailed and clubbed 
the bee nest with the side of a branch, consisting of $0.4 \%$ of tool actions. Push with foot, rotate/twist, and whisk insects were exhibited by a single juvenile, and comprised $1.1 \%, 0.2 \%$, and $0.04 \%$ of tool actions, respectively.

Most honey-gathering episodes in Goualougo involved 1 or 2 tools, but up to 5 tools were used by a single individual during a successful honey-gathering episode (Fig. 2). Although limited samples sizes prevented us from making a statistical assessment, successful tool-using episodes typically involved more tools (average $=2.14, n=14$ ) than unsuccessful honey-gathering episodes (average=1.54, $n=12$ ). Subjects did not use multiple tools simultaneously, but one after another in a serial fashion. We observed them using the same tool repeatedly during different bouts, or alternately with other tools. Most of the tools had a single function, but we also observed that a single object could be associated with several different tool-using functions (Fig. 3). Chimpanzees also frequently conserved pounding tools between bouts. Tools were placed in the canopy or held (in the foot, hand, mouth, groin pocket, or neck pocket) while the chimpanzee inspected the hive or between bouts of tool use. In Fig. 3a, we present a tool-using episode in which a chimpanzee first used a large pounding club that was eventually placed on an adjacent branch while she manufactured a smaller pounding club. After several bouts of pounding with the second club, she retrieved and reused her first tool. The female then subsequently manufactured a dipping probe to extract honey after succeeding to open the hive. This sequence of behaviors provide evidence of hierarchical structuring of tool use, with the opening task preceding the extraction task.

\section{Comparing Observations Across Sites}

The greatest diversity of tool-using behaviors in honey-gathering has been exhibited by the central subspecies of chimpanzee. In addition, pounding is almost exclusively observed within the Pan troglodytes troglodytes range, with the single exception reported from Ituri, Democratic Republic of Congo. In contrast, fluid dipping is the most commonly reported tool-using behavior in honey-gathering across sites and has been observed in all chimpanzee subspecies. The other most common tool-using
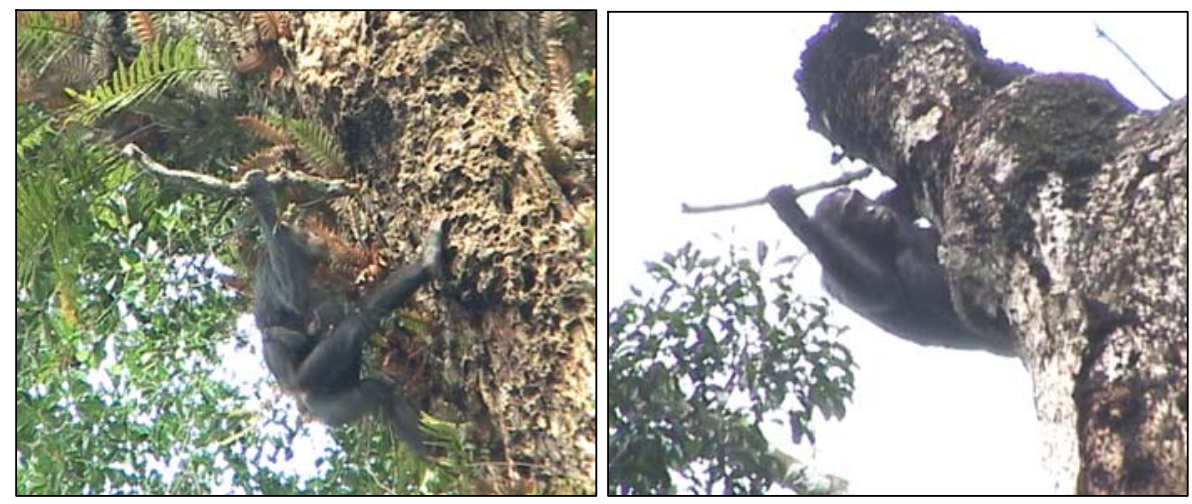

Fig. 2 Images of chimpanzees using tools to pound beehives. On the right, an adult female uses a large club to pound open a hive of Meliponini attached to the bark of a large tree. The left image shows a subadult female pounding the rotten bark of a dead tree to access a hive of Meliponini. 


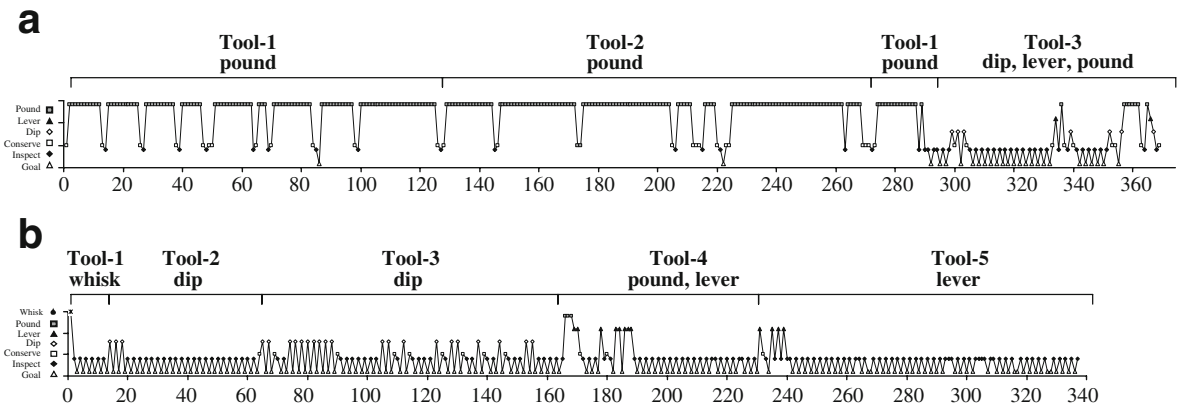

Fig. 3 Honey-gathering episodes including serial tool use of wild chimpanzees in the Goualougo Triangle, Republic of Congo. The $y$-axis summarizes behavioral elements (whisk, pound, lever, dip, conserve tool, inspect hive, and reach goal) important in achieving the honey-gathering task (Sanz and Morgan, in press) and the $x$-axis shows the progression of behavioral elements as the chimpanzee foraged for honey. (a) A tool-using episode of an adult female that used multiple tools to open a hive and then extract honey. The first 2 tools were stout branches used to pound the hive, while the third was a slender dipping probe fashioned from a twig. The first part of the episode was dominated by pounding actions that are separated by inspection of the hive. There were only 2 times that the chimpanzee consumed a small amount of honey. After the hive was opened with the reuse of the first tool, the chimpanzee manufactured a third tool to extract honey. (b) A tool using sequence of a juvenile male that first whisked away bees and then used another tool to dip for honey. The chimpanzee discarded the dipping tool and then gathered honey with his fingers, but subsequently manufactured another dipping tool. The chimpanzee then manufactured a fourth tool to pound and lever the hive entrance. The final tool was used as a lever to open the hive, after which the chimpanzee resumed eating honey.

behaviors are probing, prying/digging, and lever-opening. Researchers have attributed multiple functions to chimpanzee tools at several sites, including Lossi and Goualougo in Republic of Congo (Lossi: Bermejo and Illera 1999; Goualougo: this study) and Loango in Gabon (Boesch et al. in press).

To examine whether tool dimensions differed between tool functions, we compiled the diameter and length of tools reported from different sites. We excluded tools associated with multiple functions from this comparison. Tools used to pound and leveropen were consistently larger than tools used to dig, dip, and probe. Pounding (4.64士 $3.59 \mathrm{~cm}, n=48)$ and lever-opening $(3.40 \pm 3.14 \mathrm{~cm}, n=68)$ tools had a larger diameter than tools used to dig $(1.67 \pm 1.25 \mathrm{~cm}, n=25)$, dip $(0.98 \pm 0.37 \mathrm{~cm}, n=286)$, and probe $(0.54 \pm 0.08 \mathrm{~cm}, n=179)$. Tools used to pound $(59.99 \pm 18.60 \mathrm{~cm}, n=48)$ and leveropen $(59.83 \pm 34.67 \mathrm{~cm}, n=68)$ were longer than tools used to dig $(44.15 \pm 17.59 \mathrm{~cm}$, $n=25)$, dip $(49.93 \pm 20.54 \mathrm{~cm}, n=286)$, and probe $(25.05 \pm 14.50 \mathrm{~cm}, n=179)$. However, one should interpret these results with caution because we found evidence that terms were not used consistently to define tool behaviors across sites.

\section{Discussion}

We provide the first repeated direct observation and systematic documentation of chimpanzee tool use in honey-gathering in the Congo Basin. Researchers have observed some of these behaviors (dip) in other populations of chimpanzees, whereas others (pound/hammer) may be limited to this region. In addition, some tool 
behaviors may have been misidentified across sites owing to the lack of precise definitions of tool actions or functions. However, pounding of beehives is a distinctive behavior, with tools that surpass the size of any other honey-gathering tools used by wild chimpanzees (Table I). We confirm that pounding of beehives is habitual, defined as "seen repeatedly in several individuals, consistent with some degree of social transmission" by Whiten et al. (1999, p. 1488) among the population of wild chimpanzees in the southern portion of the Nouabalé-Ndoki National Park. Chimpanzees showed a high degree of persistence, as indicated by duration of tool using episodes and number of actions toward raiding beehives. Pounding was the most successful technique to open a hive, but chimpanzees exhibited flexible strategies and insightful problem solving in honey-gathering. We made repeated observations of sequential use of $\geq 2$ types of tools, which is a behavior pattern that is rarely observed in other wild ape populations (Fox et al. 1999; Sugiyama 1997). Although tools may initially be manufactured for a specific function, we also found that chimpanzees were capable of using a tool for multiple functions. Such complex and flexible tool using behavior has not previously been reported for chimpanzees in West or East Africa, suggesting that their may be specific factors affecting their promulgation throughout this region.

Bees of Apis sp. and Meliponini occur throughout the range of all studied chimpanzee populations. It is plausible that bee ecology or hive structures vary across the chimpanzee range, but we had the impression that each hive was structurally unique and presented a challenge to the chimpanzee targeting it. This may be due to the prevalence of mason and lodger bees that opportunistically exploit substrates to encase their hives. Such varied targets may necessitate the tool user to possess multiple tactics that can be flexibly implemented or hierarchically structured relative to the conditions of each hive encountered.

Brewer and McGrew (1990) defined the use of a tool set as the consecutive use of $>1$ type of tool for a single task. Chimpanzees in the Goualougo Triangle used multiple tools in serial order, and showed hierarchical organization of tool-using behaviors with opening tools and actions preceding those to extract honey. Brewer and McGrew (1990) reported a clear instance of serial tool use by a young female chimpanzee that used several tools to open a hive and extract honey. During their long-term field research on gorillas in Republic of Congo, Bermejo and Illera (1999) observed a chimpanzee using the opposite ends of a single tool as a chisel and a bodkin to open a beehive. Chimpanzees in the Goualougo Triangle regularly use tool sets in other foraging contexts (Sanz et al. 2004), which could increase their overall technical competence or increase the likelihood of generalizing skills between tasks. In particular, these chimpanzees seemed to have developed the technological skills of using certain tools to overcome the defenses of their prey, and then another tool to gather/extract the item. For example, a perforating twig is used to open the exit tunnels of termites on the outer surface of elevated termite nests, which provides access for an herbaceous fishing probe used to extract the termites from the inner chambers of the nest. These chimpanzees also forage for termites in subterranean nests, which requires creating a tunnel into the nest with a stout puncturing stick, and then using a fishing probe to extract the termites. We have recently documented another tool set that is used by chimpanzees in the Goualougo Triangle to forage on army ants. 
Chimpanzees in central Africa reside in sympatry with western lowland gorillas throughout most of their range. There is a high degree of dietary overlap between these species (Morgan and Sanz 2006), which may have prompted the development of advanced tool behaviors in these chimpanzees. Tool use in foraging contexts has been suggested to provide increased access to resources that results in a net energy gain for the tool user. Gunther and Boesch (1993) found a nutritional return of 9 times the energy output of nut-cracking behaviors. However, there are several indications that honey-gathering is a not a particularly beneficial foraging strategy. First, rewards for honey-gathering were variable and ranged from only a few drops of honey to several handfuls of comb. Even if bee larvae were consumed, the nutritional return of gathering wild honey, which is composed of $69-80 \%$ sugar (Roubik 1989), is not likely to provide remarkable nutritional returns for the amount of energy expended. Second, a high degree of effort was expended to open beehives and attempts were not always successful. The success rate for honey-gathering in Goualougo was only $52 \%$ for the episodes we observed, with an average of 6 tool actions required for each taste of honey. Third, there is an element of risk inherent in this tool activity, which often occurs in the high canopy. Chimpanzees suspend themselves at precarious angles to access beehives, and a juvenile fell to the ground while gathering honey on the side of a large tree. Finally, overcoming the defenses of the beehives is technically challenging and there could be costs associated with individually inventing effective tool behaviors. For example, we observed younger individuals frequently engaging in tool practice, using a detached object in an appropriate fashion toward a location that did not contain honey or the hive was impossible to open. Based on the widespread observations of hive pounding and hammering in this region, there is likely to be some social facilitation of tool techniques. We observed tool transfer and sharing of tool sites between individuals, which is similar to a previous report of chimpanzees in the Taï forest sharing a honey dipping tool (Boesch and Boesch 2000, p. 193).

Based on our literature review and field research on this topic, we identified several steps that could advance the understanding of chimpanzee tool-using behavior in honey-gathering. As demonstrated by recent reviews of chimpanzee tool use in army ant predation (Schöning et al. 2007), more systematic and coordinated sampling of bee taxa across field sites is likely to provide important information about ecological factors influencing tool use in honey-gathering. Our aim in compiling a list of tool behaviors observed in honey-gathering (Table III) was to provide an ethogram for future observations that we hope will assist in standardizing the identification of behaviors between sites. We strongly advocate that scientists record video footage of tool-using behaviors because this provides the means to review each tool using episode for detailed information that can aid in identifying or defining specific behaviors, measure interobserver reliability in identifying tool behaviors, and share the observations with other scientists. Several scientists have discussed the possibility of creating a video ethogram of tool-using behaviors for their individual site or as a collaborative effort among several sites, which would be an important resource that could greatly improve the quality of comparisons across sites.

The rich behavioral diversity and technological traditions of wild chimpanzees has important, but often overlooked conservation value. Each wild chimpanzee 
population has a distinct repertoire of tool using behaviors, and the preservation of these technological traditions should be another consideration for long-term conservation strategies (McGrew 2004). Conservation initiatives on behalf of great apes have focused on mitigating negative human impacts on ape density or distribution, but it is important to determine whether human disturbances are affecting the traditional behaviors of wild apes. Expanding human influence on wild orangutans and their behaviors prompted van Schaik (2001) to propose "The Disturbance Hypothesis for the Loss of Local Traditions in Orangutans," which suggests that local extinction, hunting pressure, logging, and habitat loss may disrupt the transmission processes of traditional behaviors among wild apes. Initial studies of chimpanzees in the Congo Basin have expanded our understanding of the behavioral diversity of this species, and future research promises even more discoveries that will be possible only if we ensure the long-term preservation of these great apes and their habitats.

Acknowledgments We thank the Ministry of Forest Economy of the Republic of Congo and the Wildlife Conservation Society for their support and collaboration. We also recognize the tireless dedication of J. R. Onononga, C. Eyana-Ayina, S. Ndolo, and the Goualougo tracking team. Special thanks are due to J. M. Fay, F. Maisels, B. Curran, P. Elkan, S. Elkan, E. Stokes, M. Gately, H. Thomas, and B. Dos Santos. R. Mundry provided assistance with statistical analysis. D. Roubik kindly identified bee specimens from this site and reviewed our compilation of bee taxa reported from other sites. This manuscript was greatly improved by discussions with $\mathrm{C}$. Boesch and E. Lonsdorf. We also thank T. C. Hicks for his careful review of this manuscript. C. Boesch also generously provided information about tool dimensions and functions from Loango, Gabon. We thank the U. S. Fish and Wildlife Service, National Geographic Society, Wildlife Conservation Society, Columbus Zoological Park, Lowry Park Zoo, Brevard Zoological Park, and Great Ape Trust of Iowa for funding this research.

Open Access This article is distributed under the terms of the Creative Commons Attribution Noncommercial License which permits any noncommercial use, distribution, and reproduction in any medium, provided the original author(s) and source are credited.

\section{References}

Bermejo, M., \& Illera, G. (1999). Tool-set for termite-fishing and honey extraction by wild chimpanzees in the Lossi Forest, Congo. Primates, 40(4), 619-627. doi:10.1007/BF02574837.

Bermejo, M., Illera, G., \& Sabater-Pi, J. (1989). New observations on the tool-behavior of chimpanzees from Mt. Assirik (Senegal, West Africa). Primates, 30(1), 65-73. doi:10.1007/BF02381211.

Boesch, C., \& Boesch, H. (1990). Tool use and tool making in wild chimpanzees. Folia Primatologica, 54, 86-99. doi:10.1159/000156428.

Boesch, C., \& Boesch-Achermann, H. (2000). The chimpanzees of the Tai Forest: Behavioural ecology and evolution. Oxford: Oxford University Press.

Boesch, C., Head, J., \& Robbins, M. M. Complex toolsets for honey extraction among chimpanzees in Loango National Park, Gabon. Journal of Human Evolution, in press.

Brewer, S. M., \& McGrew, W. C. (1990). Chimpanzee use of tool set to get honey. Folia Primatologica, 54, 100-104. doi:10.1159/000156429.

Byrne, R. W., \& Byrne, J. M. E. (1993). Complex leaf-gathering skills of mountain gorillas Gorilla $g$. beringei: Variability and standardization. American Journal of Primatology, 31, 241-261. doi:10.1002/ajp.1350310402.

Byrne, R. W., Corp, N., \& Byrne, J. M. E. (2001). Estimating the complexity of animal behaviour: How mountain gorillas eat thistles. Behaviour, 138, 525-557. doi:10.1163/156853901750382142.

Deblauwe, I. (2006). New evidence of honey-stick use by chimpanzees in southeast Cameroon. Pan African News, 13, 2-4. 
Fay, J. M., \& Carroll, R. W. (1994). Chimpanzee tool use for honey and termite extraction in central Africa. American Journal of Primatology, 33, 309-317. doi:10.1002/ajp.1350340403.

Fleiss, J. L. (1981). Statistical methods for rates and proportions (2nd ed.). New York: John Wiley \& Sons.

Fowler, A., \& Sommer, V. (2007). Subsistence technology of Nigerian chimpanzees. International Journal of Primatology, 28(5), 997-1023. doi:10.1007/s10764-007-9166-0.

Fox, E. A., Sitompul, A. F., \& van Schaik, C. P. (1999). Intelligent tool use in wild Sumatran orangutans. In S. T. Parker, R. W. Mitchell, \& H. L. Miles (Eds.), The mentalities of gorillas and orangutans: Comparative perspectives (pp. 99-116). Cambridge, UK: Cambridge University Press.

Goodall, J. (1968). The behaviour of free-living chimpanzees in the Gombe Stream Reserve. Animal Behaviour Monographs, 1, 161-311.

Goodall, J. (1986). The chimpanzees of Gombe: Patterns of behavior. Cambridge, MA: Belknap Press.

Gunther, M., \& Boesch, C. (1993). Energetic costs of nut-cracking behavior in wild chimpanzees. In D. Chivers, \& H. Preuschoft (Eds.), Evolution of hands (pp. 109-129). Stuttgart: Gustav Fisher Verlag.

Hicks, T. C., Fouts, R. S., \& Fouts, D. H. (2005). Chimpanzee (Pan troglodytes troglodytes) tool use in the Ngotto Forest, Central African Republic. American Journal of Primatology, 65, 221-237. doi:10.1002/ajp.20111.

Izawa, I., \& Itani, J. (1966). Chimpanzees in the Kasakati Basin, Tanganyika. 1. Ecological study of the rainy season. Kyoto University African Studies, 1, 73-156.

Kajobe, R., \& Roubik, D. W. (2006). Honey-making bee colony abundance and predation by apes and humans in a Uganda forest reserve. Biotropica, 38(2), 210-218. doi:10.1111/j.1744-7429.2006.00126.x.

Mangold (2006). INTERACT Software, version 8.04. Germany: Arnstorf.

McGrew, W. C. (1992). Chimpanzee material culture: Implications for human evolution. Cambridge, UK: Cambridge University Press.

McGrew, W. C. (2004). The cultured chimpanzee: Reflections on cultural primatology. Cambridge, UK: Cambridge University Press.

Merfield, F. G., \& Miller, H. (1956). Gorillas were my neighbours. London: Longmans, Green and Co.

Morgan, D., \& Sanz, C. (2006). Chimpanzee feeding ecology and comparisons with sympatric gorillas in the Goualougo Triangle, Republic of Congo. In G. Hohmann, M. Robbins, \& C. Boesch (Eds.), Primate feeding ecology in apes and other primates: Ecological, physiological, and behavioural aspects (pp. 97-122). Cambridge, UK: Cambridge University Press.

Nishida, T., \& Hiraiwa, M. (1982). Natural history of a tool-using behavior by wild chimpanzees in feeding upon wood-boring ants. Journal of Human Evolution, 11(1), 73-99. doi:10.1016/S0047-2484(82)80033-X.

Nishida, T., \& Uehara, S. (1983). Natural diet of chimpanzees (Pan troglodytes schweinfurthii): Long-term record from the Mahale Mountains, Tanzania. African Study Monographs, 3, 109-130.

Ohashi, G. (2006). Behavioral repertoire of tool use in the wild chimpanzees at Bossou. In T. Matsuzawa, M. Tomonaga, \& M. Tanaka (Eds.), Cognitive development in chimpanzees (pp. 439-451). Tokyo: Springer-Verlag.

Roubik, D. W. (1989). Ecology and natural history of tropical bees. Cambridge: Cambridge University Press.

Sanz, C., \& Morgan, D. (2007). Chimpanzee tool technology in the Goualougo Triangle, Republic of Congo. Journal of Human Evolution, 52, 420-433. doi:10.1016/j.jhevol.2006.11.001.

Sanz, C., \& Morgan, D.(in press). Complexity of chimpanzee tool using behaviors. In E. V. Lonsdorf, S. R. Ross, \& T. Matsuzawa. The mind of the chimpanzee: Ecological and empirical perspectives. Chicago, Il: University of Chicago Press.

Sanz, C., Morgan, D., \& Gulick, S. (2004). New insights into chimpanzees, tools, and termites from the Congo Basin. American Naturalist, 164, 567-581. doi:10.1086/424803.

Schoning, C., Humle, T., Mobius, Y., \& McGrew, W. C. (2007). The nature of culture: Technological variation in chimpanzee predation on army ants revisited. Journal of Human Evolution, 55(1), 48-59. doi:10.1016/j.jhevol.2007.12.002.

Stanford, C. B., Gambaneza, C., Nkurunungi, J. B., \& Goldsmith, M. L. (2000). Chimpanzees in BwindiImpenetrable National Park, Uganda, use different tools to obtain different types of honey. Primates, 41(3), 337-341. doi:10.1007/BF02557602.

Sugiyama, Y. (1997). Social tradition and the use of tool-composites by wild chimpanzees. Evolutionary Anthropology, 6, 23-27. doi:10.1002/(SICI)1520-6505(1997)6:1<23::AID-EVAN7>3.0.CO;2-X.

Tutin, C. E. G., Ham, R., \& Wrogemann, D. (1995). Tool-use by chimpanzees (Pan t. troglodytes) in the Lopé Reserve, Gabon. Primates, 36(2), 181-192. doi:10.1007/BF02381344.

van Schaik, C. P. (2001). Fragility of traditions: The disturbance hypothesis for the loss of local traditions in orangutans. International Journal of Primatology, 23(3), 527-538. doi:10.1023/A:1014965516127. 
Watts, D. (2008). Tool use by chimpanzees at Ngogo, Kibale National Park, Uganda. International Journal of Primatology, 29, 83-94. doi:10.1007/s10764-007-9227-4.

Whiten, A., Goodall, J., McGrew, W. C., Nishida, T., Reynolds, V., Sugiyama, Y., et al. (1999). Cultures in chimpanzees. Nature, 399, 681-685.

Whiten, A., Goodall, J., McGrew, W. C., Nishida, T., Reynolds, V., Sugiyama, Y., Tutin, C. E. G., Wrangham, R., \& Boesch, C. (2001). Charting cultural variation in chimpanzees. Behaviour, 138, 1481-1516. doi:10.1163/156853901317367717.

Wrangham, R. W. (1975). The behavioural ecology of chimpanzees in Gombe National Park, Tanzania. Ph.D. dissertation. Cambridge University, U.K.

Yamagiwa, J., Yumoto, T., Ndunda, M., \& Maruhashi, T. (1988). Evidence of tool-use by chimpanzees (Pan troglodytes schweinfurthii) for digging out a bee-nest in the Kahuzi-Biega National Park, Zaire. Primates, 29(3), 405-411. doi:10.1007/BF02380963.

Yamakoshi, G., \& Myowa-Yamakoshi, M. (2004). New observations of ant-dipping techniques in wild chimpanzees at Bossou, Guinea. Primates, 45, 25-32. doi:10.1007/s10329-003-0056-6. 